\title{
Editorial
}

\section{Solar Energy Conversion by Nanostructured $\mathrm{TiO}_{2}$}

\author{
Jia Hong Pan, ${ }^{1}$ Detlef W. Bahnemann, ${ }^{1}$ Qing Wang, ${ }^{2}$ Chuanyi Wang, ${ }^{3}$ and Xiwang Zhang ${ }^{4}$ \\ ${ }^{1}$ Institut für Technische Chemie, Leibniz Universität Hannover, Callinstraße 3, 30167 Hannover, Germany \\ ${ }^{2}$ Department of Materials Science \& Engineering, Faculty of Engineering, National University of Singapore, \\ 5 Engineering Drive 2, Singapore 117576 \\ ${ }^{3}$ Laboratory of Eco-Materials and Sustainable Technology (LEMST), Xinjiang Technical Institute of Physics and \\ Chemistry and Key Laboratory of Functional Materials and Devices for Special Environments, Chinese Academy of Sciences, \\ Urumqi, Xinjiang 830011, China \\ ${ }^{4}$ Department of Chemical Engineering, Monash University, Clayton, VIC 3800, Australia
}

Correspondence should be addressed to Jia Hong Pan; pan@iftc.uni-hannover.de

Received 24 August 2014; Accepted 24 August 2014; Published 22 December 2014

Copyright (C) 2014 Jia Hong Pan et al. This is an open access article distributed under the Creative Commons Attribution License, which permits unrestricted use, distribution, and reproduction in any medium, provided the original work is properly cited.

Research in solar energy conversion and the associated photoactive materials has attracted continuous interest. Due to its proper electronic band structure, high quantum efficiency, and photonic and chemical innerness, $\mathrm{TiO}_{2}$ has been demonstrated as a versatile oxide semiconductor capable of efficiently utilizing sunlight to produce electrical and chemical energy. Its outstanding physicochemical performances have led to an array of advanced photocatalytic and photoelectrochemical applications including environmental photocatalysis, dye/semiconductor-sensitized solar cell, and solar fuel productions.

Scientific papers in this special issue have covered this highly developing field. Authors have submitted review and original research articles in the following topics:

(i) kinetics and mechanism of $\mathrm{TiO}_{2}$-mediated environmental photocatalysis,

(ii) $\mathrm{TiO}_{2}$-based dye/semiconductor-sensitized solar cells,

(iii) photocatalytic organic synthesis over $\mathrm{TiO}_{2}$-mediated system,

(iv) photocatalytic solar fuel production (water splitting and $\mathrm{CO}_{2}$ reduction),

(v) novel $\mathrm{TiO}_{2}$-based nanomaterials for solar energy conversion.

We wish to express our sincere gratefulness to all the authors and referees whose contributions make this special issue possible. A brief overview of all nine accepted papers is below.

In "Recent progress in $\mathrm{TiO}_{2}$-mediated solar photocatalysis for industrial wastewater treatment," the authors overview the major challenges in industrial wastewater treatment and analysed the merits of $\mathrm{TiO}_{2}$ photocatalysis over conventional water treatment technologies. Then a brief but comprehensive review is carried out on the recent progress in their applications in several typical industrial wastewaters. The paper could advance the development of solar photocatalysis systems and promote their practical application.

In "Photocatalytic degradation of anthracene in closed system reactor," the authors investigate the effect of operating parameters on the degradation efficiency of anthracene, one of the toxic persistent organic pollutants. The photodegradation products are identified by GC-MS to better understand the degradation path of anthracene on $\mathrm{P} 25 \mathrm{TiO}_{2}$ nanoparticles under UV irradiation. Optimum parameters have been systematically investigated to obtain a high photocatalytic degradation rate. The research reported here gives an excellent example how to design proper photocatalytic process for organic pollutants removal.

In "Sol-gel to prepare nitrogen doped $\mathrm{TiO}_{2}$ nanocrystals with exposed $\{001\}$ facets and high visible-light photocatalytic performance," a facile synthetic method based on sol-gel process is developed to synthesize anatase $\mathrm{TiO}_{2}$ with dominant $\{001\}$ facets and nitrogen doping by simply hydrolysing tetrabutyl titanate in $\mathrm{NH}_{4} \mathrm{~F}$-containing alcoholic solution. $\mathrm{NH}_{4} \mathrm{~F}$ 
not only acts as the $\mathrm{N}$ dopant source, but also guides the preferential growth along [001] direction. The synthesized $\mathrm{N}$-doped $\mathrm{TiO}_{2}$ nanoparticles outperform P25 referents in photocatalytic degradation of methylene blue under visible light irradiation.

In "One-dimensional nanostructured $\mathrm{TiO}_{2}$ for photocatalytic degradation of organic pollutants in wastewater," current progress in the synthetic methods for one-dimensional (1D) $\mathrm{TiO}_{2}$ nanostructures including nanorods, nanotubes, nanowires/nanofibers, and nanobelts is reviewed. Modification of $1 \mathrm{D} \mathrm{TiO}_{2}$ with metal oxide, metal ions, and anions in order to enhance the photocatalytic activity is discussed. Furthermore, photocatalytic degradations of organic pollutants in wastewater over $1 \mathrm{D} \mathrm{TiO}_{2}$ are summarized, and the underlying mechanism is discussed. Finally, using 1D nanostructured $\mathrm{TiO}_{2}$ as building blocks to construct film or membrane is highlighted.

In "Layer-by-layer assembly and photocatalytic activity of titania nanosheets on coal fly ash microspheres," an attempt to address the problem with nanoparticulate $\mathrm{TiO}_{2}$ distribution and recovery is made. Coal fly ash (CFA) microspheres are used as a substrate for the layer-by-layer assembly of $\mathrm{Ti}_{0.91} \mathrm{O}_{2}$ sheets. That is, the $\mathrm{Ti}_{0.91} \mathrm{O}_{2}$ nanosheets are immobilized on CFA by using sequential modification of cationic polyelectrolyte and $\mathrm{Ti}_{0.91} \mathrm{O}_{2}$ nanosheets. The resultant $\mathrm{Ti}_{0.91} \mathrm{O}_{2} / \mathrm{CFA}$ composites show considerable photocatalytic activity in degradation of methylene blue under UV irradiation. After photocatalysis, the $\mathrm{Ti}_{0.91} \mathrm{O}_{2} / \mathrm{CFA}$ can be easily separated and recycled from aqueous solution.

In "Effect of $M n$ doping on properties of CdS quantum dot-sensitized solar cells," impurity $\mathrm{Mn}^{2+}$ ions are doped into the precursor solution for CdS deposition. By optimizing the experimental parameters, a significant improvement in photoelectric conversion efficiency can be achieved. After a successive ionic layer adsorption and reaction of a fixed number of six times, the photoelectric conversion efficiency shows the maximum value $(1.51 \%)$ at the optimal doped ratio, which is much higher than that of the pure one $(0.71 \%)$.

In "Theoretical study of one-intermediate band quantum dot solar cell," the authors theoretically studied the influence of the new band on the power conversion efficiency for the structure of the quantum dots of intermediate band solar cell. The time-independent Schrödinger equation is used to determine the optimum width and location of the intermediate band. From their calculation results, the maximum power conversion efficiency is about $70.42 \%$ for simple cubic quantum dot crystal under full concentration light. It is strongly dependent on the width of quantum dots and barrier distances.

In "Equilibrium and kinetic aspects in the sensitization of monolayer transparent $\mathrm{TiO}_{2}$ thin films with porphyrin dyes for DSSC applications," the adsorption of free base and $\mathrm{Cu}$ (II) and $\mathrm{Zn}$ (II) complexes of the 2,7,12,17-tetrapropionic acid of 3,8,13,18-tetramethyl-21H,23H porphyrin (CPI) on transparent monolayer $\mathrm{TiO}_{2}$ nanoparticle films was studied. The dye loading is found to be accordant with Langmuir isotherm, while kinetic data show significantly better fits to pseudofirst-order model and the evaluated rate constants linearly increase with the grow of initial dye concentrations. The stoichiometry of the adsorption of CPI-dyes into $\mathrm{TiO}_{2}$ and the influence of presence of coadsorbent (chenodeoxycholic acid) are established. This study paves a way for choosing the best experimental conditions for the adsorption of these dyes.

In "Steric and solvent effect in dye-sensitized solar cells utilizing phenothiazine-based dyes," three novel phenothiazinebased dyes are prepared and utilized for dye-sensitized solar cells (DSSC). The dye-bath solvent presents a significant effect on the overall cell performance. The highest conversion efficiency of $3.78 \%$ is obtained using ethanol (EtOH) and $2.53 \%$ for tetrahydrofuran (THF) as solvent, respectively. The higher efficiency is related to the higher dye loading and coverage on the $\mathrm{TiO}_{2}$ surface. Meanwhile, phenothiazine-based dyes with longer and branched aliphatic chain increase the steric hindered effect which is mainly responsible for increasing the electron lifetime and decreases the dye aggregation as well as increasing the electron recombination resistance rate at the $\mathrm{TiO}_{2}$-dye-electrolyte interface, hence, enhancing the overall cell performance.

Jia Hong Pan

Detlef W. Bahnemann Qing Wang Chuanyi Wang Xiwang Zhang 

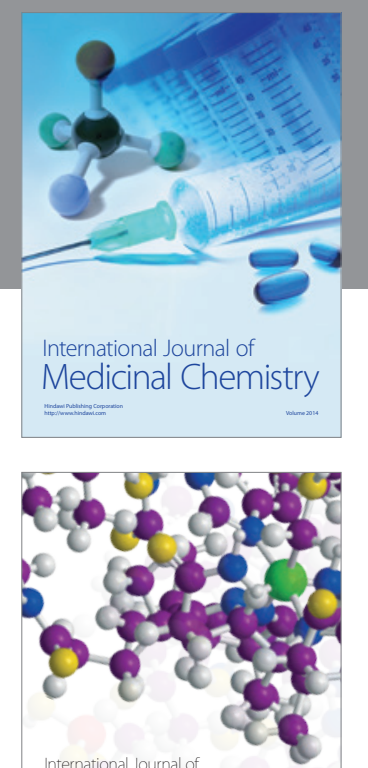

\section{Carbohydrate} Chemistry

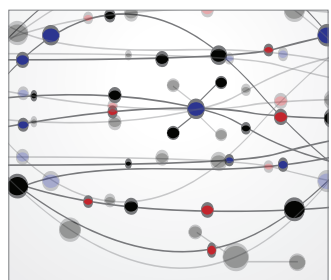

The Scientific World Journal
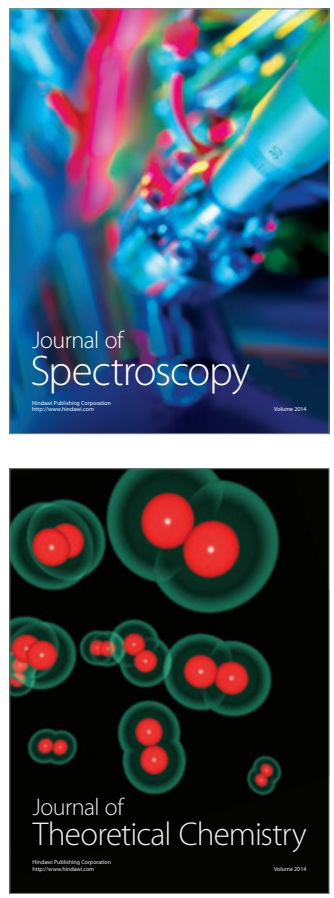
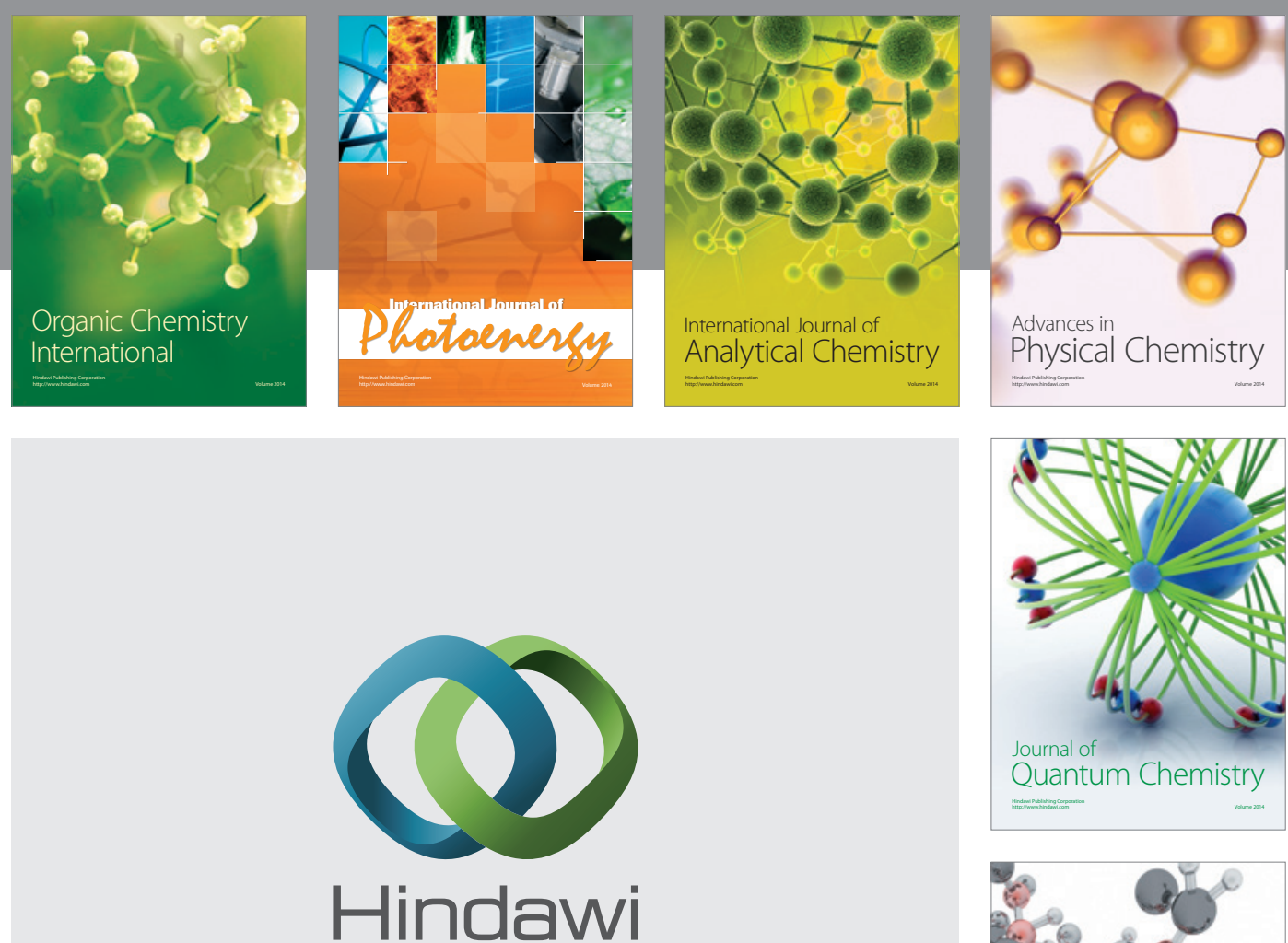

Submit your manuscripts at

http://www.hindawi.com

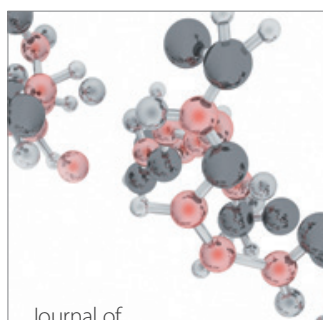

Analytical Methods

in Chemistry

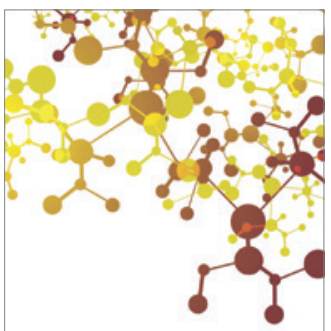

Journal of

Applied Chemistry

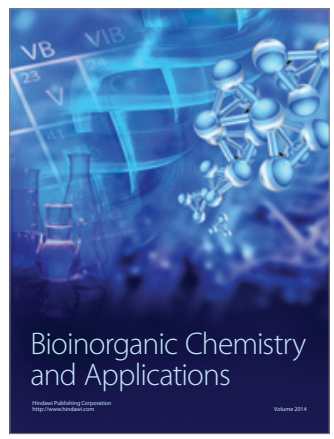

Inorganic Chemistry
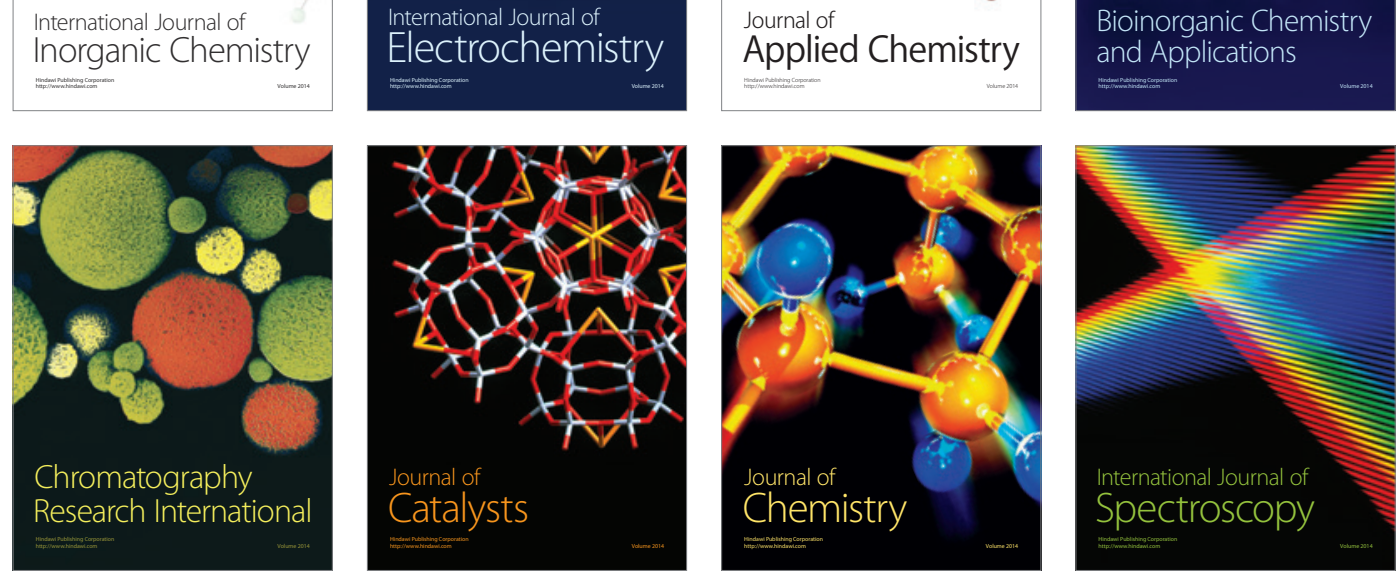